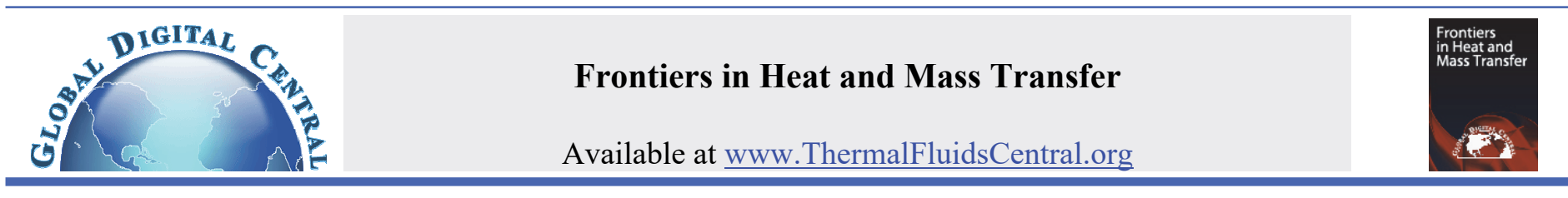

\title{
EFFECTS OF VARIABLE FLUID PROPERTIES ON A DOUBLE DIFFUSIVE MIXED CONVECTION VISCOUS FLUID OVER A SEMI INFINITE VERTICAL SURFACE IN A SPARSELY PACKED MEDIUM
}

\author{
R. Suresh Babu ${ }^{1,2}$, B. Rushi Kumar ${ }^{1 *}$ and P.A. Dinesh ${ }^{2}$ \\ ${ }^{1}$ Department of Mathematics, School of Advanced Sciences, Vellore Institute of Technology, Vellore - 632014, India \\ ${ }^{2}$ Department of Mathematics, Ramaiah Institute of Technology, Bangalore - 560 054, India
}

\begin{abstract}
This problem deals with the effects of double diffusive, mixed convective flow of an incompressible viscous fluid through a vertical heated plate embedded in a non-Darcy porous medium under the influence of variable fluid properties numerically. The governing equations are modeled for the double diffusive boundary layer flow to understand the behaviour of velocity, temperature and concentration for variable fluid properties namely permeability, porosity, thermal conductivity and solutal diffusivity of the physical modal. Using a suitable similarity transformation, the highly nonlinear coupled PDE's are reduced into a set of coupled ordinary differential equations. By applying the Shooting technique with the help of RungeKutta-Fehlberg integral scheme and Newton-Raphson method, numerical computations have been carried out and are illustrated through the graphs to analyze the behaviour of velocity, temperature, concentration, Skin friction, Nusselt number and Sherwood numbers for the non-dimensional parameters of the physical system for both uniform permeability and variable permeability. To validate our numerical scheme, present results are compared with previously published work in the absence of few non-dimensional parameters and found to be in good agreement up to six decimal places of accuracy.

Keywords: Double diffusive, Forchheimer model, Mixed convection, Variable fluid properties, Shooting technique.
\end{abstract}

\section{INTRODUCTION}

Double diffusive convective fluid flow have been analyzed by several authors in a porous medium over the last four decades with many physical parameters for different geometries, because such study has a wide range of practical applications in an industrial as well as engineering fields. For instance, dispersion of chemical reactions in chemical industry, petroleum industry, casting and welding in producing process, packed-bed reactors, drying of porous solids, geothermal extraction, storage of waste material, thermal insulation engineering, cooling of electronic components etc. Also many natural applications in the environment like distribution of heat and wetness over farming fields and groves trees, soil pollution, crystal growth dispersion of dissolved materials, evaporation of a water body at the surface, damage of crops due to freezing, dehydration and drying operations in chemical, food processing plants etc. Comprehensive theories, practical applications, experiments of thermal convection, review of literature in porous media have been reported by Nield and Bejan (2013).

In literature, many researchers while investigating the behaviour of the fluid, especially the fluid properties namely the permeability, porosity, thermal conductivity and solutal diffusivity of the porous medium are taken as constants. But most of the realistic situations insists of the physical properties with variable characteristics because the fluid properties are prone to vary with a temperature and concentration gradients, especially in the boundary layer theory. The variation of porosity of the porous regime has been first studied by incorporated the variable permeability concept and proved that, the variations of porosity and permeability has a maximum influence on the fluid as well as on heat transfer through and past a porous medium.

Hong et al. (1987) have studied the effect of non-uniform porosity and non-Darcian flow on a vertical heated plate in porous media. Using local similarity solution, Kafoussias (1990) studied the effect of mixed convective mass transfer flow through the vertical plate. Mehta and Rao (1994) have been examined non-uniform surface heat flux of nonNewtonian fluids with buoyancy-induced flow in a porous medium. Hady et al.(1996) studied the effect of variable viscosity on free and forced convective boundary layer flow for a continuous flat plate in a porous medium.

The effect of variable permeability and porosity on convective flow from the vertical surface were analyzed numerically first by Mohammadein and El-Shaer (2004). Seddeek (2004) illustrated the thermal-diffusion, diffusion thermo and variable viscosity effects through an accelerating surface along a internal heat generation in the occurrence of suction and blowing. The inertial effect is more important for high speed flows and it can be calculated by adding an additional velocity term to the momentum equation is called as Forchheimer Darcy law. Pal and Shivakumara (2006) examined the influence of variable permeability and porosity on mixed convection of a non-Darcy MHD fluid flow through a vertical plate embedded in a porous medium. Numerical computations has been carried out by Chamkha et al.(2007) on mixed convective, heat and mass transfer flow of a non-Newtonian 
fluid for a permeable surface in a porous medium and compared with analytical work. Mahesha Narayana et al. (2009) analyzed the powerlaw fluid over a vertical stretching sheet by considering variable thermal conductivity and non-uniform heat source/sink, assuming that the thermal conductivity vary linearly with temperature. Satya Narayana and Sravanthi (2012) studied the radiation absorption and chemical reactions of an unsteady mixed convective flow for an incompressible electrically conducting fluid through a porous medium between two vertical channels in the presence of heat generation. Harish Babu and Satya Narayana (2013) investigated an extensive study on unsteady free convective heat and mass transfer flow for a micropolar fluid with a variable permeability bounded by a semiinfinite vertical plate in the occurrence of heat generation/ absorption, thermal radiation, first-order chemical reaction in a porous medium. Rushi Kumar and Shivaraj (2013) have been studied the effects of variable viscosity and viscous dissipation in thermal boundary layer fluid flow as a function of temperature over a vertical cone and flat plate along with MHD flow in heat and mass transfer. Nalinakshi et al (2013) have been discussed variable fluid properties for a vertical plate by incorporating the magnetic field in a porous medium. Satya Narayana (2015) studied effects of variable permeability and radiation absorption on MHD mixed convective flow in a vertical wavy channel with traveling thermal waves numerically. Srinivasacharya et al.(2015) analyzed the flow behaviour on a sinusoidal wavy surface through a porous medium numerically by taking variable fluid properties for a Darcy model. Venkateswarlu and Satya Narayana (2016) discussed about the influence of non uniform thermal conductivity variations on MHD caisson fluid flow with viscous dissipation, Soret and Dufour effects on a stretching sheet.

Keeping the above discussions in mind, authors investigated to explore the effect of double diffusive, mixed convective, heat and mass transfer flow of a Forchheimer model through a vertical plate in a porous medium under the influence of variable fluid properties. The non-linear coupled PDE's are reduced into a system of ODE's using similarity transformation, then the resultant BVP is solved numerically using shooting technique to analyze the flow pattern of velocity, temperature, concentration, Skin friction, rate of heat and mass transfers for various physical parameters. Here we discussed both the uniform and variable fluid properties against each non-dimensional parameter of the physical system.

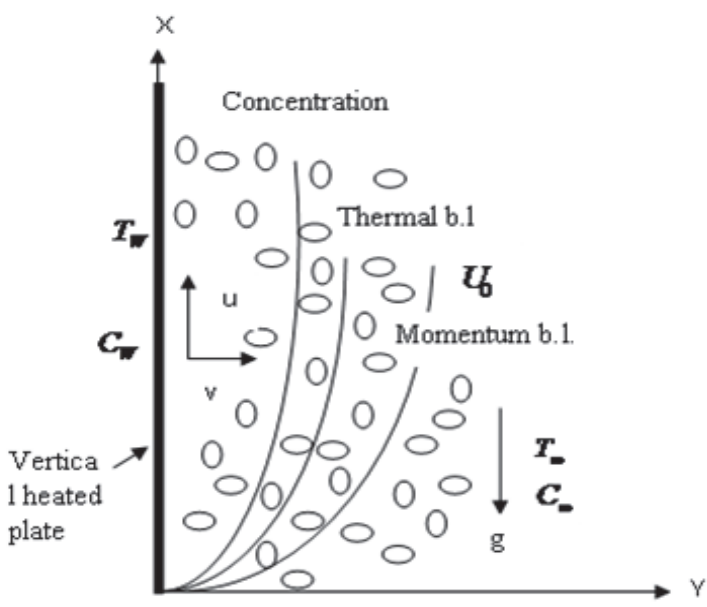

Fig. 1 Physical configaration

\section{MATHEMATICAL FORMULATION}

Consider a steady, two-dimensional, laminar, double diffusive, mixed convection, heat and mass transfer flow of an incompressible viscous fluid over a vertical surface in a sparsely packed porous medium. The flow configuration of the physical model is shown in Fig. 1 and the coordinate system is chosen as follows. Here, the $x$ - axis is taken along the plate from one of its edge and $y$ - axis is taken exactly perpendicular to the plate. Let $U_{0}$ be the free stream velocity in the upward direction and assumed to be constant along the vertical surface and $g$ is the gravitational force acting towards down. The plate is maintained at a uniform temperature $T_{w}$, uniform concentration $C_{w}$ which vary with free stream fluid temperature $T_{\infty}$ and concentration $C_{\infty}$, where the plate is considered to be heated $T_{w}>T_{\infty}$ (Assisting flow) and cooled $T_{w}<T_{\infty}$ (Opposing flow).

The Forchheimer model is used to explain the flow characteristics of the porous medium due to the low permeability and assumed that the flow is moderate. The coordinate system is assumed to be locally hydrodynamic and thermal equilibrium between the fluid and solid phase. Under these assumptions by invoking Boussinesq and boundary layer approximations, the basic governing equations are modeled as follows

$$
\begin{aligned}
& \frac{\partial u}{\partial x}+\frac{\partial u}{\partial x}=0, \\
& u \frac{\partial u}{\partial x}+v \frac{\partial u}{\partial x}=-\frac{1}{\rho} \frac{\partial p}{\partial x}+g \beta_{T}\left(T-T_{\infty}\right)-g \beta_{C}\left(C-C_{\infty}\right)+\frac{\bar{\mu}}{\rho} \frac{\partial^{2} u}{\partial y^{2}} \\
& -\frac{\mu}{\rho} \frac{\varepsilon(y)}{k(y)} u-C_{b} \frac{\varepsilon^{2}(y)}{\sqrt{k(y)}} u^{2} \\
& u \frac{\partial T}{\partial x}+v \frac{\partial T}{\partial y}=\frac{\partial}{\partial y}\left(\alpha(y) \frac{\partial T}{\partial y}\right)+\frac{\bar{\mu}}{\rho C_{p}}\left(\frac{\partial u}{\partial y}\right)^{2}, \\
& u \frac{\partial C}{\partial x}+v \frac{\partial C}{\partial y}=\frac{\partial}{\partial y}\left(\gamma(y) \frac{\partial C}{\partial y}\right)
\end{aligned}
$$

The associated boundary conditions are

$$
\begin{array}{ll}
u=0 \quad: v=0, T=T_{w}, C=C_{w} & \text { at } y=0, \\
u=U_{0}: v=0, T=T_{\infty}, C=C_{\infty} & \text { as } y \rightarrow 0 .
\end{array}
$$

Here, we introduced the stream function $\psi(x, y)$ to satisfy the continuity equation in the dimensionless form, we may write

$u=\frac{\partial \psi}{\partial y}, v=\frac{-\partial \psi}{\partial y}$

Where the components of velocity along the $x$ and $y$ axis respectively are given by

$u=U_{0} f^{\prime}(\eta), v=-\frac{1}{2} \sqrt{\frac{v U_{0}}{x}}\left(f(\eta)-\eta f^{\prime}(\eta)\right)$.

The following local similarity transformation is used to reduce the above PDE's (2), (3) and (4) into ODE's

$\psi=\sqrt{v U_{0} x} f(\eta)$; where $\eta=\frac{y}{x}\left(\frac{U_{0} x}{v}\right)^{1 / 2}$.

Also, the variable fluid properties namely permeability $k(\eta)$, porosity $\varepsilon(\eta)$, thermal conductivity $\alpha(\eta)$ and solutal diffusivity $\gamma(\eta)$ are assumed to be as

$$
\begin{aligned}
& k(\eta)=k_{0}\left(1+d e^{-\eta}\right) ; \varepsilon(\eta)=\varepsilon_{0}\left(1+d^{*} e^{-\eta}\right) \\
& \alpha(\eta)=\alpha_{0}\left(\varepsilon_{0}\left(1+d^{*} e^{-\eta}\right)+\sigma^{*}\left\{1-\varepsilon_{0}\left(1+d^{*} e^{-\eta}\right)\right\}\right)
\end{aligned}
$$




$$
\gamma(\eta)=\gamma_{0}\left(\varepsilon_{0}\left(1+d^{*} e^{-\eta}\right)+\gamma^{*}\left\{1-\varepsilon_{0}\left(1+d^{*} e^{-\eta}\right)\right\}\right) .
$$

where $k_{0}, \varepsilon_{0}, \alpha_{0}$ and $\gamma_{0}$ are the initial amplitudes of permeability, porosity, thermal conductivity and solutal diffusivity of the porous medium and $\sigma^{*}$ is the ratio of thermal conductivity of solid to the fluid, $\gamma^{*}$ is the ratio of solutal diffusivity of solid to the fluid. Here, $d$ and $d^{*}$ are assumed as initial amplitudes of Uniform permeability (UP) and Variable permeability (VP) and their values are defined as in [ Mohammadein and El-Shaer, Pal and Shivakumara and Nalinakshi et al. ]. Rewriting equations (2), (3) and (4) by using equations(7), (8) and (9), we get

$$
\begin{aligned}
& f^{\prime \prime \prime}+\frac{1}{2} f f^{\prime}+R i(\theta-\phi)+\frac{1}{D a \operatorname{Re}}\left(\frac{1+d^{*} e^{-\eta}}{1+d e^{-\eta}}\right)\left(1-f^{\prime}\right) \\
& +\frac{F s}{D a} \frac{\left(1+d^{*} e^{-\eta}\right)^{2}}{\left(1+d e^{-\eta}\right)^{1 / 2}}\left(1-f^{\prime 2}\right)=0 \\
& \theta^{\prime \prime}=\frac{\left(\frac{1}{2} \operatorname{Pr} f \theta^{\prime}+\operatorname{Pr} E f^{\prime \prime 2}+\varepsilon_{0} d^{*} e^{-\eta}\left(\sigma^{*}-1\right) \theta^{\prime}\right)}{\varepsilon_{0}+\sigma^{*}\left(1-\varepsilon_{0}\right)+\varepsilon_{0} d^{*} e^{-\eta}\left(1-\sigma^{*}\right)}, \\
& \phi^{\prime \prime}=\frac{\left(\frac{1}{2} S c f \phi^{\prime}+\varepsilon_{0} d^{*} e^{-\eta}\left(\gamma^{*}-1\right) \phi^{\prime}\right)}{\varepsilon_{0+\gamma^{*}}\left(1-\varepsilon_{0}\right)+\varepsilon_{0} d^{*} e^{-\eta}\left(1-\gamma^{*}\right)} .
\end{aligned}
$$

Where $R i=\frac{G r}{\mathrm{Re}^{2}}$ is the Richardson number, $\mathrm{Re}=\frac{U_{0} x}{v}$ is the local Reynolds number, $D a=\frac{k_{p}}{x^{2}}$ is the Darcy number, $F s=\frac{b}{x}$ is the Forchheimer number, $\operatorname{Pr}=\frac{\bar{\mu}}{\rho \alpha_{0}}$ is the Prandtl number, $\sigma^{*}=\frac{k_{0}}{x^{2} \varepsilon_{0}}$ is the local permeability parameter, $S c=\frac{\bar{\mu}}{\rho v_{0}}$ is the Schmidt number, $N=\frac{\beta_{C}\left(C_{w}-C_{\infty}\right)}{\beta_{T}\left(T_{w}-T_{\infty}\right)}$ is the Buoyancy ratio, $E=\frac{U_{0}{ }^{2}}{C_{p}\left(T_{w}-T_{\infty}\right)}$ is the Eckert number.

The boundary conditions (5) in terms of $f, \theta$ and $\phi$ becomes

$$
\begin{array}{r}
f=0, f^{\prime}=0, \theta=1, \phi=1 \text { at } \eta=0 . \\
f^{\prime}=1, \theta=0, \phi=0 \text { as } \eta \rightarrow \infty .
\end{array}
$$

The Skin friction, rate of heat and mass transfers for the vertical plate are given by $\tau=-f^{\prime \prime}(0) / \sqrt{\operatorname{Re}}$,

$q_{w}=-k\left(\frac{\partial T}{\partial y}\right)_{y=0}$ and $q_{m}=-D\left(\frac{\partial C}{\partial y}\right)_{y=0}$

The non-dimensional local Nusselt number $N u=\frac{x q_{w}}{k\left(T_{w}-T_{\infty}\right)}$,

$$
\begin{aligned}
& \text { Sherwood numbers } \quad S h=\frac{x q_{m}}{D\left(C_{w}-C_{\infty}\right)} \quad \text { are given by } \\
& N u=-\theta^{\prime}(0) \sqrt{\operatorname{Re}}, S h=-\phi^{\prime}(0) \sqrt{\operatorname{Re}} \text {. }
\end{aligned}
$$

\section{METHOD OF SOLUTION}

The flow Eqs.(10)-(12) are solved along with its boundary conditions (13) by using shooting technique with the help of $4^{\text {th }}$ order R-K method and Newton-Raphson algorithm numerically. The fundamental idea of this method for the BVP is, to find an appropriate initial condition where computed solution hit the target. This method has four major steps. They are (i) higher order non-linear differential equations are reduced into a system of simultaneous linear differential equations of first order; (ii) transform BVP into IVP by applying shooting technique; (iii) the resultant IVP is solved by employing Runge-Kutta fourth order integral technique; (iv) then choose appropriate initial guess values with the help of Newton-Raphson method where the boundary conditions will be satisfied. The iterative procedure is repeated until the error becomes less than a predefined accuracy level. In the present study, the value of $\eta$ is to be chosen sufficiently large at each time, where the velocity profile approaches to one, the temperature and concentration profiles are approaches to zero at the outer edge of the boundary layer. To validate our results, a numerical comparison has done in each case and found that in good agreement.

\section{RESULTS AND DISCUSSION}

Numerically based computational work is invoked for the study of variable fluid property effects on double diffusive, mixed convection for viscous fluid over a vertical surface in a porous medium. The system of non-linear coupled ODE's having variable coefficients are solved for non-dimensional velocity, temperature and concentration by shooting technique. An in detailed numerical study has been performed for the above said flow having non-dimensional parameters like Mixed convection parameter, Forchheimer number, Prandtl number Schmidt number, Thermal conductivity parameter and Solutal diffusivity parameter by keeping the other parameters as constants for both UP and VP cases. To validate our numerical scheme, present results are compared with earlier works published by Mohammadein and El-Shaer in the absence of buoyancy ratio, inertial parameter and concentration distribution which is shown in Table-1 for UP and VP cases. Also, comparison has been made with Nalinakshi et al. in the absence of Soret and Dufour numbers for VP case and an excellent agreement is observed up to six decimal places of accuracy but comparisons are not shown here for want of space.

Table-1: Comparison of $f^{\prime \prime}(0)$ and $-\theta^{\prime}(0)$ for VP case.

\begin{tabular}{ccccccc}
\hline$\sigma^{*}$ & $R i$ & $\alpha^{*} / \sigma \operatorname{Re}$ & \multicolumn{2}{c}{ Present result } & \multicolumn{2}{c}{ Mhd. and El-Shaer } \\
\hline & & & $f^{\prime \prime}(0)$ & $-\theta^{\prime}(0)$ & $f^{\prime \prime}(0)$ & $-\theta^{\prime}(0)$ \\
\hline $\mathbf{2}$ & $\mathbf{0 . 2}$ & $\mathbf{0 . 0}$ & 0.61215 & 0.38031 & 0.61215 & 0.38030 \\
2 & 0.2 & $\mathbf{0 . 1}$ & 0.64526 & 0.38281 & 0.64526 & 0.38281 \\
2 & 0.2 & $\mathbf{0 . 5}$ & 0.75526 & 0.38959 & 0.75527 & 0.38959 \\
2 & 0.5 & 0.0 & 0.95816 & 0.40308 & 0.95816 & 0.40308 \\
2 & $\mathbf{0 . 5}$ & 0.1 & 0.97433 & 0.40323 & 0.97432 & 0.40325 \\
2 & $\mathbf{2 . 0}$ & 0.0 & 2.31559 & 0.40378 & 2.31558 & 0.40376 \\
4 & 0.2 & $\mathbf{0 . 1}$ & 0.65771 & 0.50664 & 0.65772 & 0.50664 \\
$\mathbf{6}$ & 0.5 & 0.1 & 1.00406 & 0.52939 & 1.00403 & 0.52940 \\
\hline
\end{tabular}

\subsection{Effect of Mixed convention parameter $(R i)$}

In Figs. 2-4, the velocity, temperature and concentration profiles are illustrated for distinct values of Mixed convention parameter $R i$. From Fig. 2, as $R i$ raises the dimensionless velocity increases for both UP and VP cases because the moment of the free convection currents which are induced by the free constant stream velocity moves away from the plate to the free stream regime in the downward direction of the plate, so that the induced mean velocity of the fluid increases in both UP and VP cases. From Figs. 3 and 4, an opposite behaviour is seen in the case 
of temperature and concentration profiles as $R i$ increases in both UP and VP cases. This is due to the fact that, the induced mean velocity drives the flow which intern transfer the heat and species concentration to a lower level. Also we observed that VP dominates over UP.

\subsection{Effect of Forchheimer number $(F s)$}

The characteristics of the fluid for different values of Forchheimer number $F s$ are discussed in Figs. 5-7. Fig. 5 depicts the decrease of velocity profile with an augment of $F s$ due to fact that, the drag experienced by the porous medium dominates over the viscosity of the flow. To our knowledge in the literature similar effect has been seen on velocity for the Forchheimer term in the momentum equation as a particular case. With this velocity behaviour on $F s$, the boundary layer thickness of temperature and concentration decreases which results an enhancement characteristics on temperature and concentration shown in Figs. 6 and 7. Here UP dominates VP in the case of velocity, whereas, VP dominates UP in the case of temperature and concentration profiles.

\subsection{Effect of Prandtl number (Pr)}

The effect of Prandtl number Pr is shown in Figs. 8-10 on the nondimensional velocity, temperature and concentration profiles. From Fig. 8, it is seen that there is a significant reduction in the velocity profile with an enhancement of Pr. This is due to the higher viscosity present in the fluid flow as compared to any other forces. Similar kind of behaviour is seen in the temperature from Fig. 9 and an opposite behaviour is observed in concentration profile from Fig. 10 due to fact that, the thermal diffusivity decreases with respect to the viscosity of the fluid. The effect of $\operatorname{Pr}$ is significantly seen in velocity and temperature compared to that of concentration profiles.

\subsection{Effect of Schmidt number $(S c)$}

The velocity and concentration profiles for distinct values of Schmidt number $S_{c}$ are depicted in Figs. 11-12. An enhancement in the magnitude of $S c$ raises the velocity of the flow is observed in Fig. 11 because as thinning of the boundary layer thickness for concentration. An opposite behavior is observed in concentration distributions as increasing in $S_{c}$ from Figs. 12 due to the fact that, the solutal diffusivity decreases with respect to the viscosity of the fluid flow. The effect of Schmidt number is very less in the case of temperature profiles.

\subsection{Effects of Thermal conductivity and Solutal diffusivity parameters $\left(\sigma^{*} \& \gamma^{*}\right)$}

These effects are raised due to introducing the variable fluid properties on thermal and solutal diffusivities. Here we made an attempt to understand the effect of $\sigma^{*}$ and $\gamma^{*}$ on velocity, temperature and concentration which is shown in Figs. 13-16. The variation of Thermal conductivity parameter $\sigma^{*}$ on velocity and temperature are depicted in Figs. $13 \& 14$ and observed that the variation of $\sigma^{*}$ enhances the velocity as well as temperature profiles due to fact that the variable fluid property one can achieve higher moment of the fluid flow as well as heat exchange between the plate to the free stream regime. Similarly the effect of $\gamma^{*}$ on velocity and concentration are depicted in Figs. 15 \& 16. With a positive change of variable diffusivity parameter decreases the moment of the fluid and enhances for concentration of the fluid due to the density of the solutal fluid increases which intern deduces the velocity of the moment of the fluid in both UP and VP cases.

Also numerical study has been done for the skin-friction coefficient, Nusselt number and Sherwood number are analyzed through the graphs from Figs. 17-25. The non-dimensional skin friction, heat and mass transfer rates are depicted as a function of Richardson number $R i$ in both UP and VP cases respectively. The effects of Schmidt number $S c$ are depicted in Figs. 17-19. Enchasing the value of $S c$, there is a decay in the local skin friction coefficient, heat transfer rate but an opposite behaviour is seen in the case of mass transfer for both UP and VP cases. In Fig. 20 the variation of local skin-friction coefficient is shown and is observed that the skin-friction coefficient decrease with increasing the value of $\sigma^{*}$, also can seen that UP is higher than VP case. The variation of heat and mass transfers are presented in Figs. 21 and 22 and found that the increase with enhancing the value of $\sigma^{*}$ and VP is higher than UP. The characteristics of local skin-friction coefficient, heat and mass transfer rates on $\gamma^{*}$ are examined in Figs. 23-25 for both cases. From Figs. 23 and 24, it is seen that the skin friction coefficient and heat transfer rate enhances with enhancing the values of $\gamma^{*}$ in both cases and UP dominates VP. But the rate of mass transfer decreases as increasing the values of $\gamma^{*}$ is observed from Fig. 25.

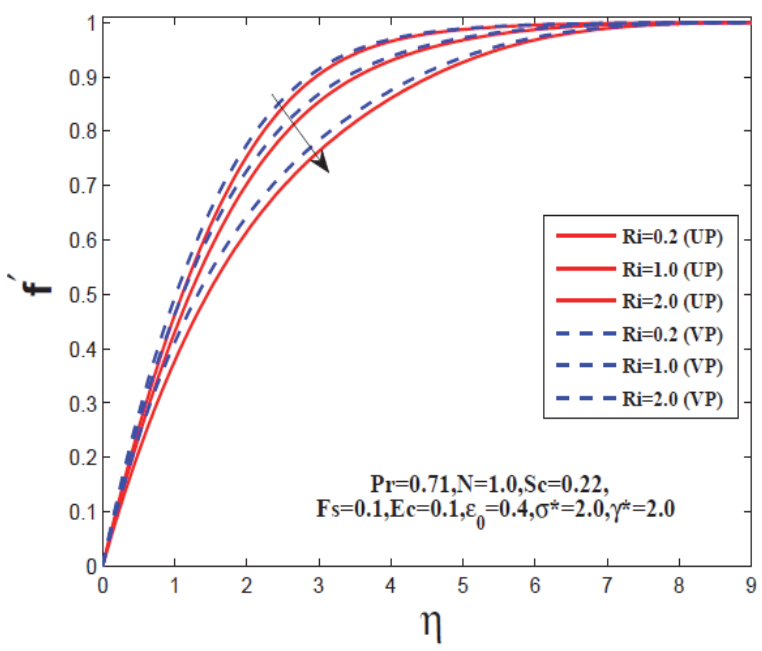

Fig. 2 Effect of $R i$ on velocity profiles.

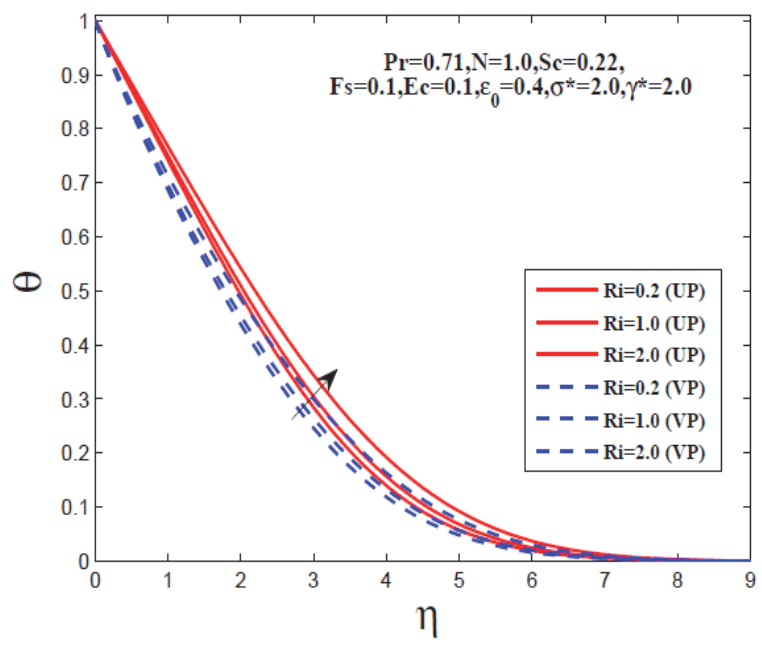

Fig. 3 Effect of $R i$ on temperature profiles. 


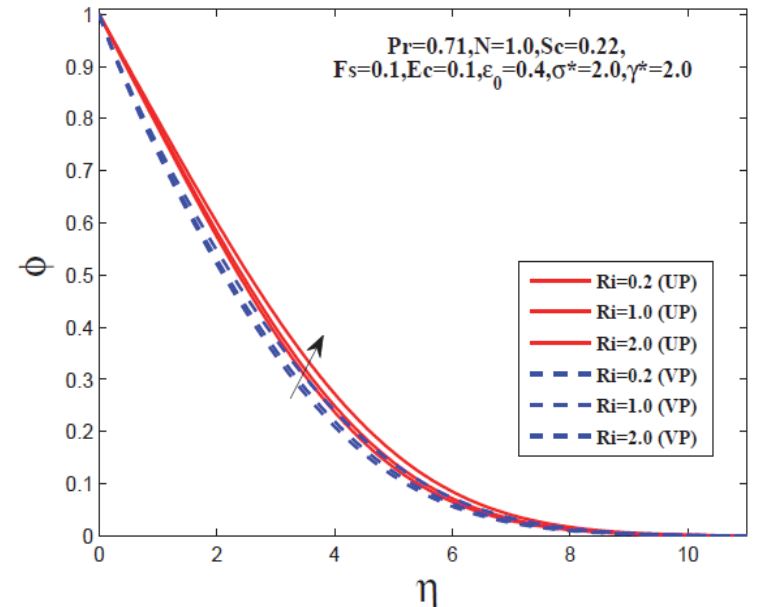

Fig. 4 Effect of $R i$ on concentration profiles.

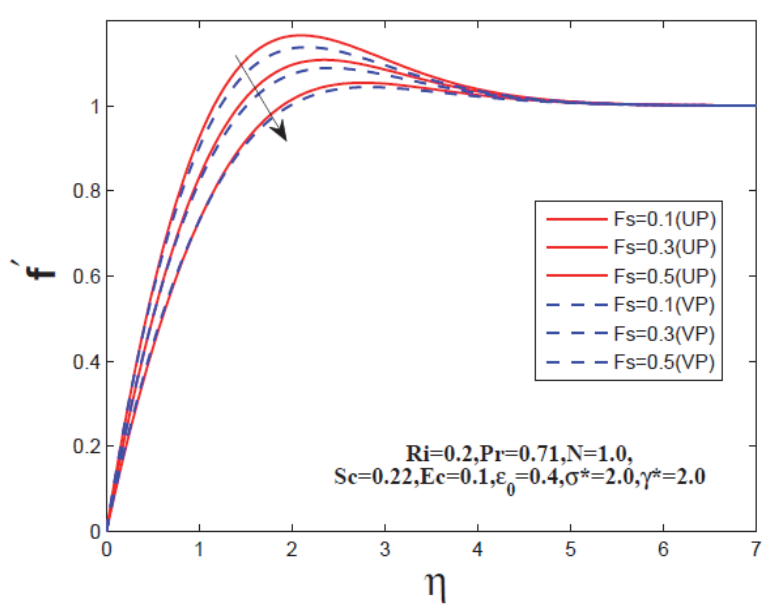

Fig. 5 Effect of $F s$ on velocity profiles.

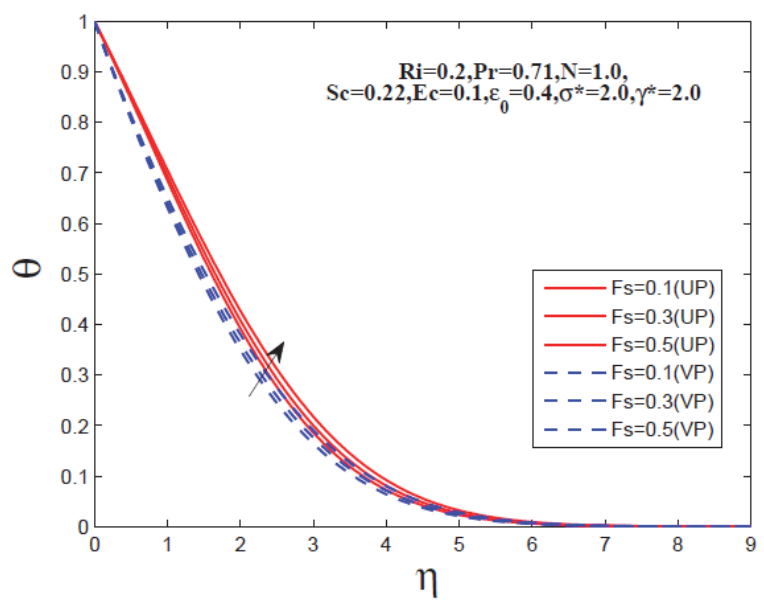

Fig. 6 Effect of Fs on temperature profiles.

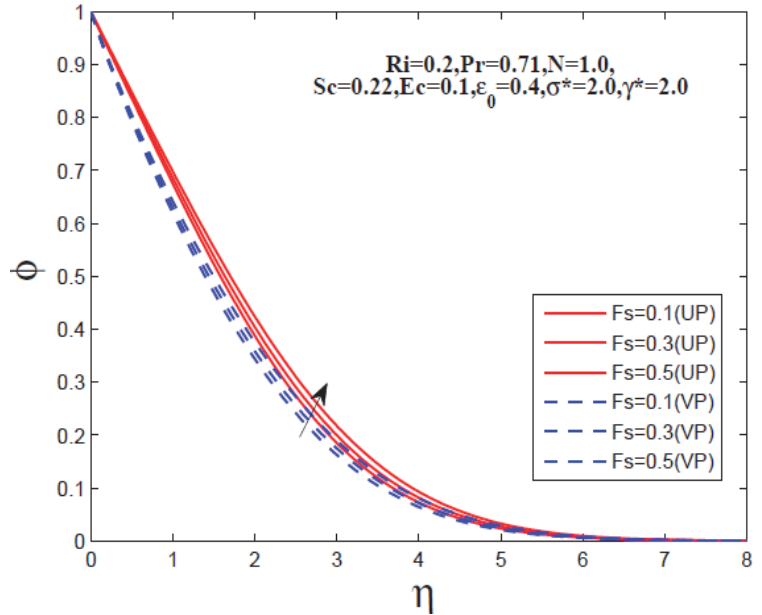

Fig. 7 Effect of $F s$ on concentration profiles.

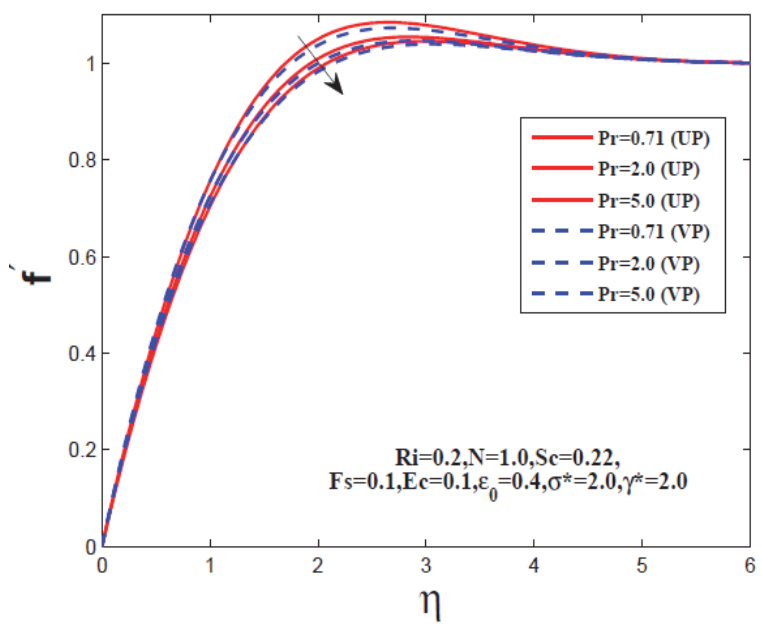

Fig. 8 Effect of Pron velocity profiles.

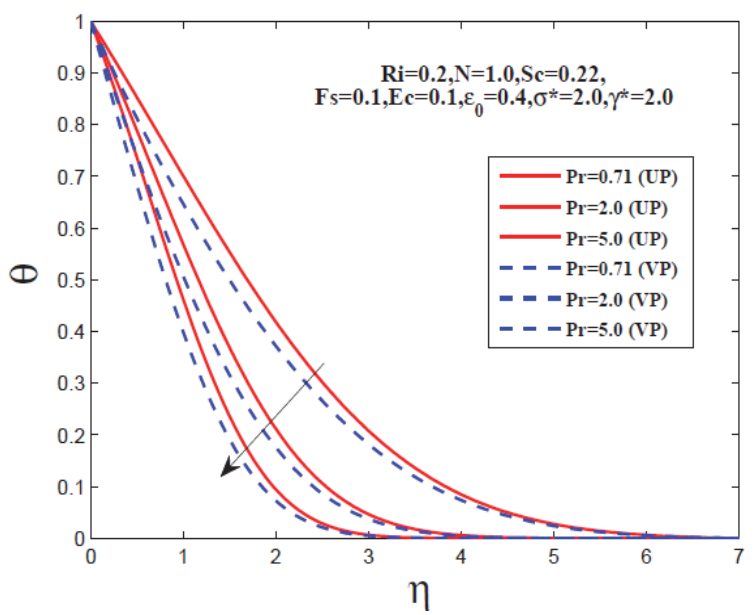

Fig. 9 Effect of Pr on temperature profiles. 


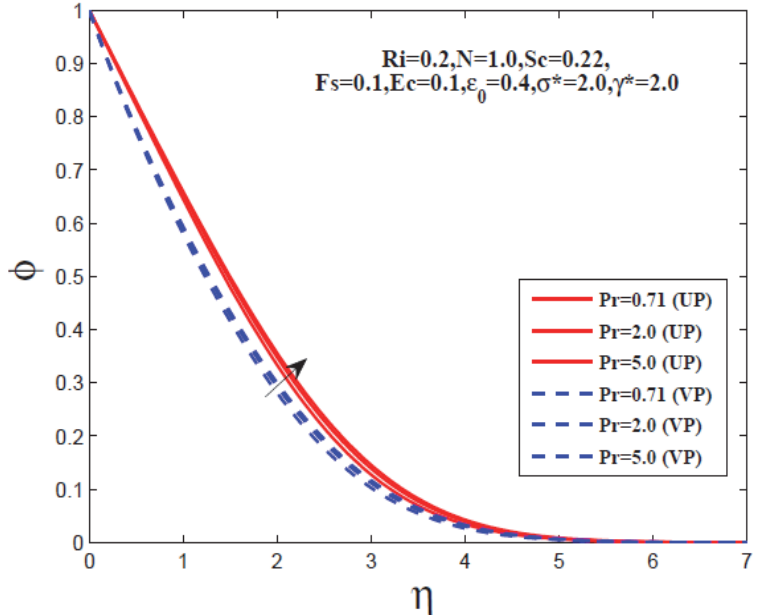

Fig. 10 Effect of Pron concentration profiles.

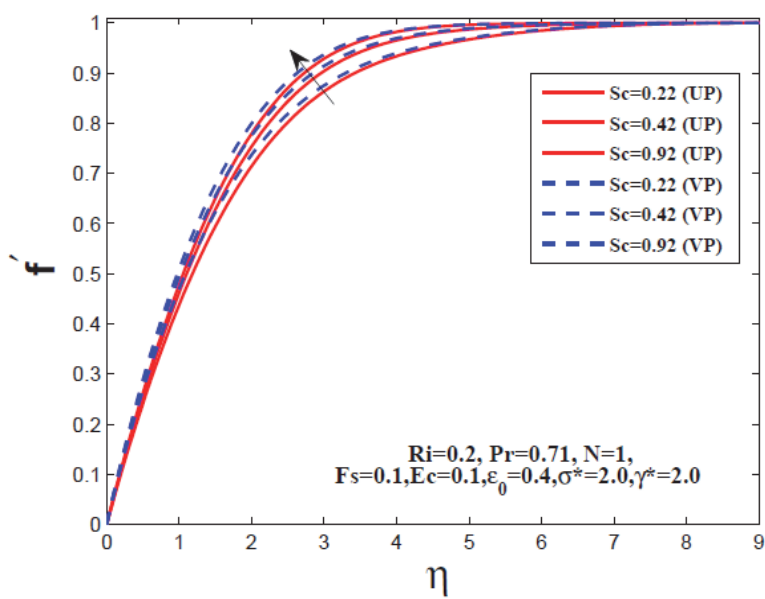

Fig. 11 Effect of $S c$ on velocity profiles.

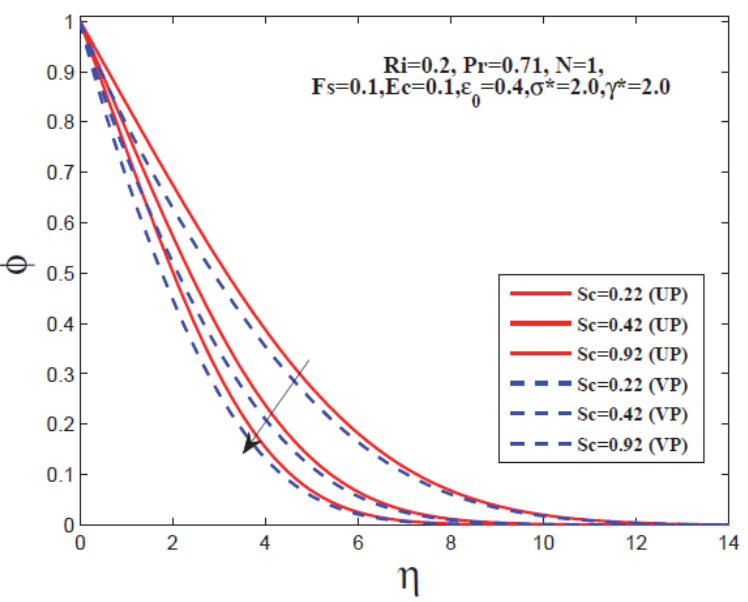

Fig. 12 Effect of $S c$ on Concentration profiles.

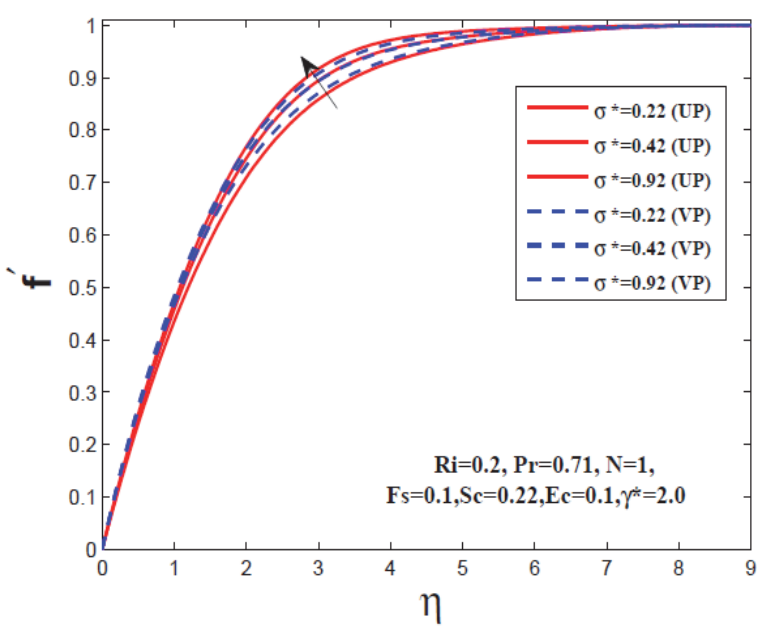

Fig. 13 Effect of $\sigma^{*}$ on Velocity profiles.

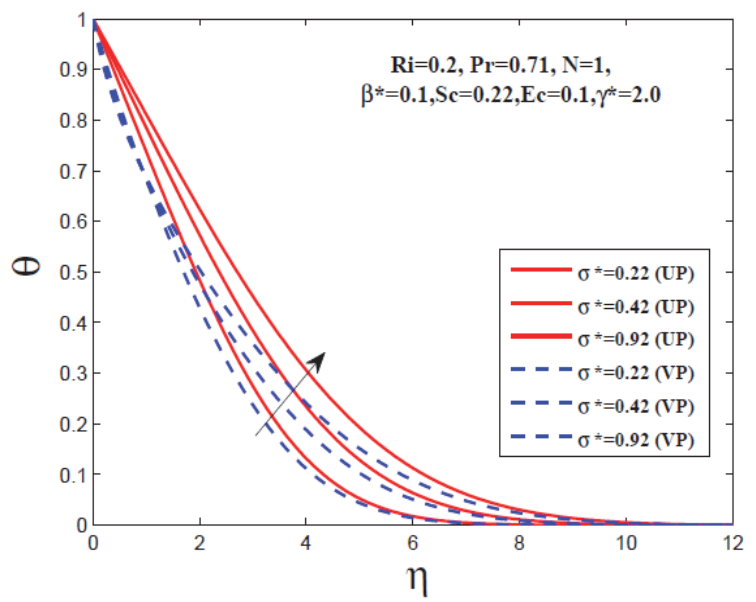

Fig. 15 Effect of $\sigma^{*}$ on temperature profiles.

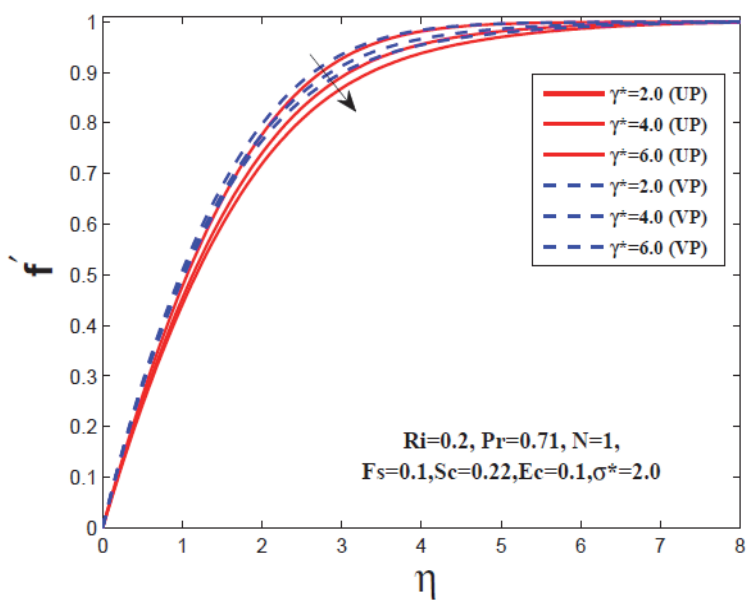

Fig. 16 Effect of $\gamma^{*}$ on Velocity profiles. 


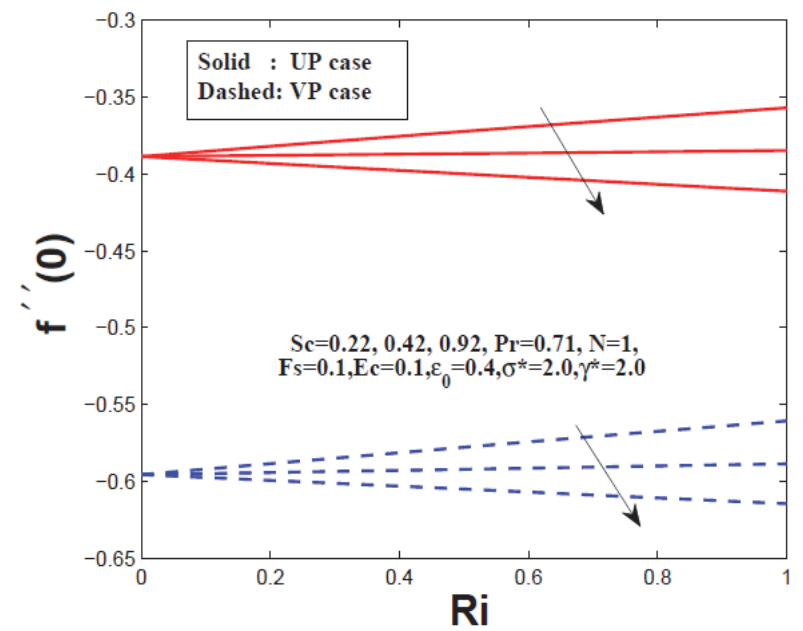

Fig. 17 Effect of Sc on skin friction.

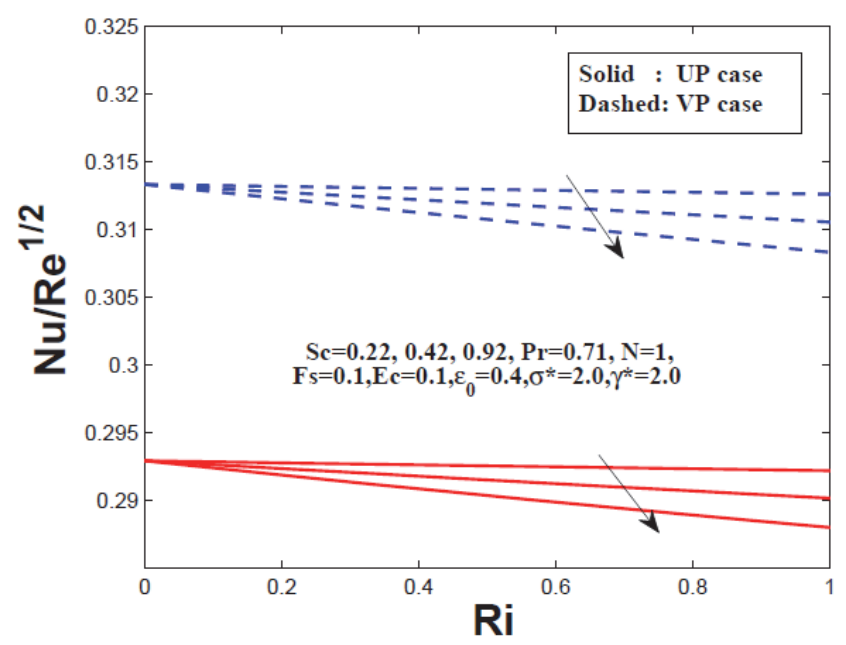

Fig. 18 Effect of $S c$ on heat transfer.

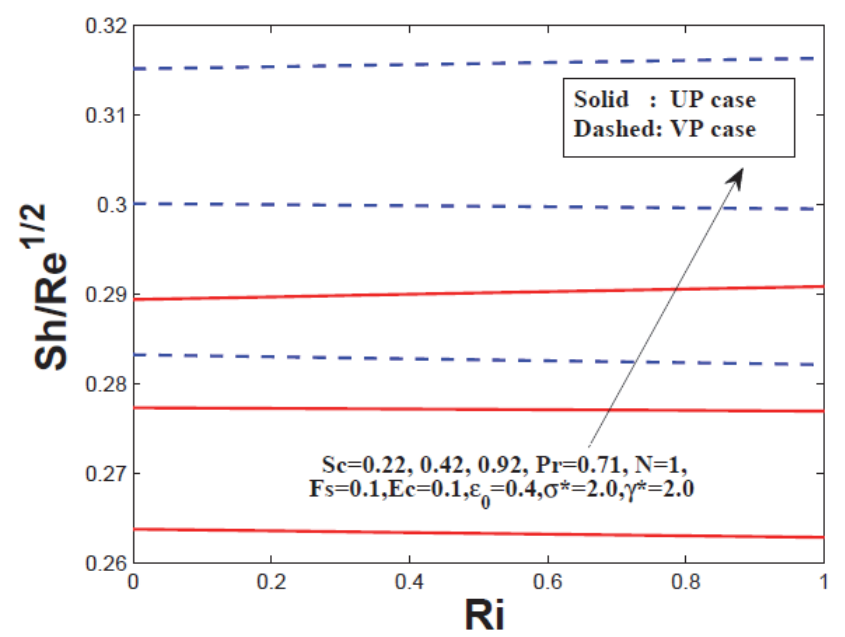

Fig. 19 Effect of $S c$ on mass transfer.

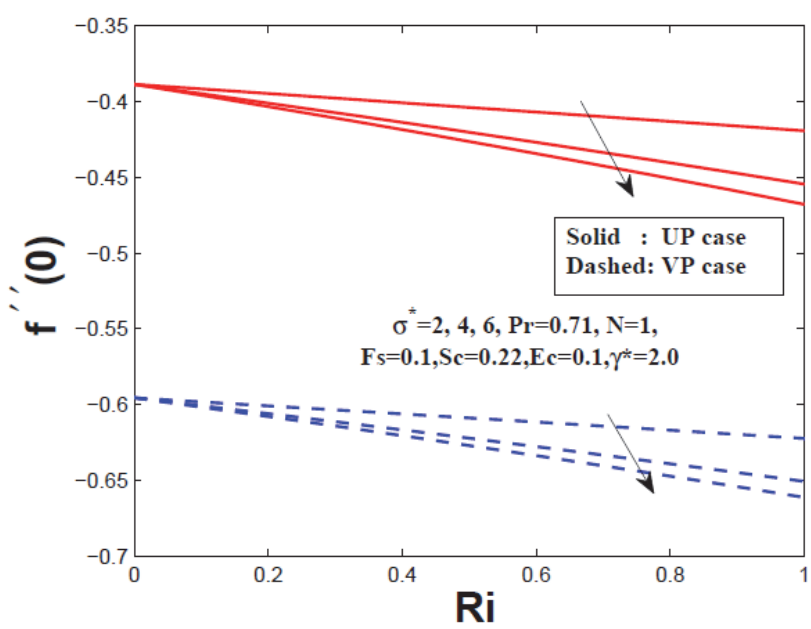

Fig. 20 Effect of $\sigma^{*}$ on skin friction.

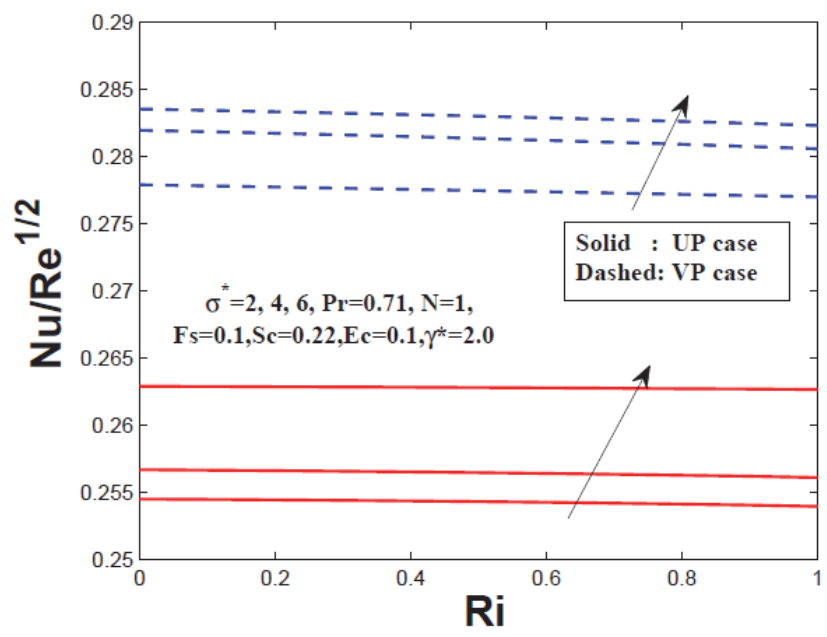

Fig. 21 Effect of $\sigma^{*}$ on heat transfer.

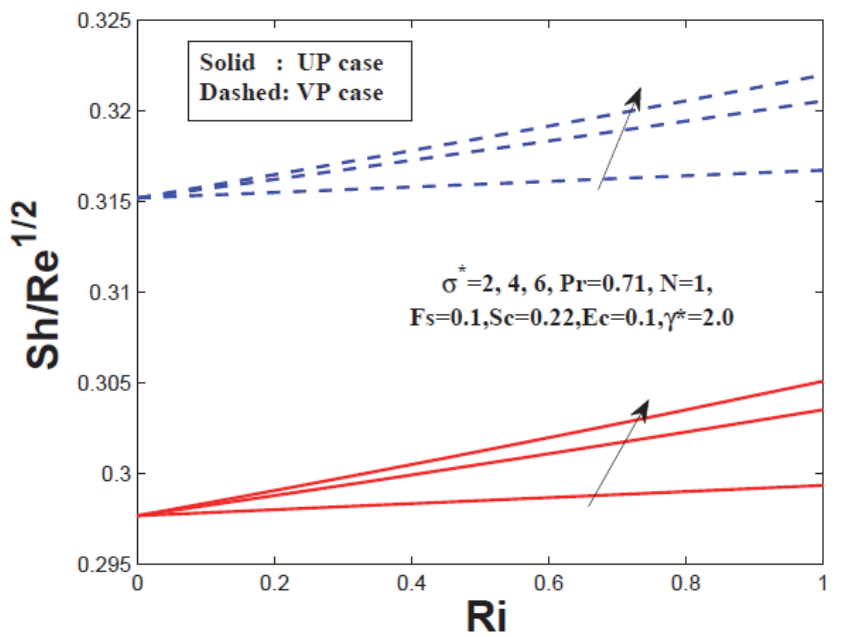

Fig. 22 Effect of $\sigma^{*}$ on mass transfer. 


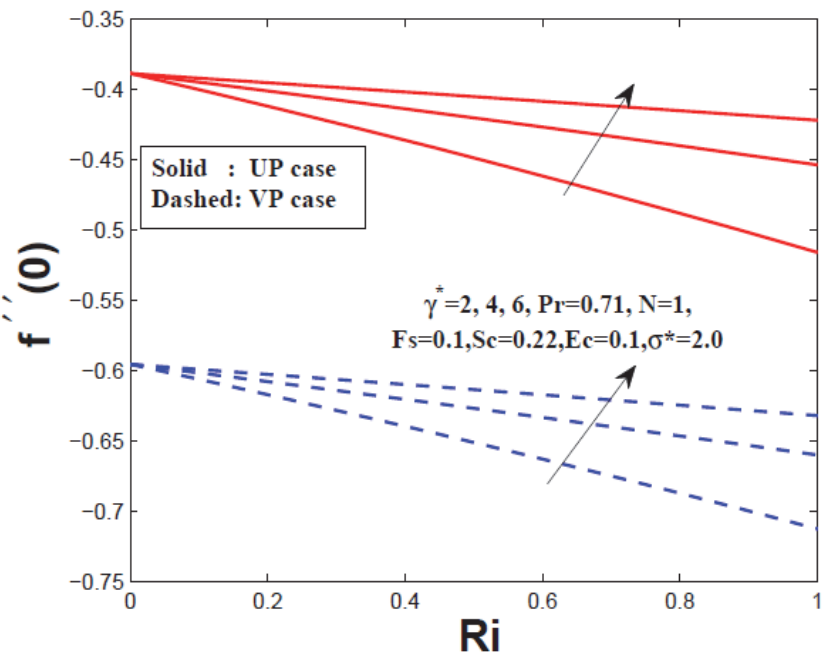

Fig. 23 Effect of $\gamma^{*}$ on skin friction.

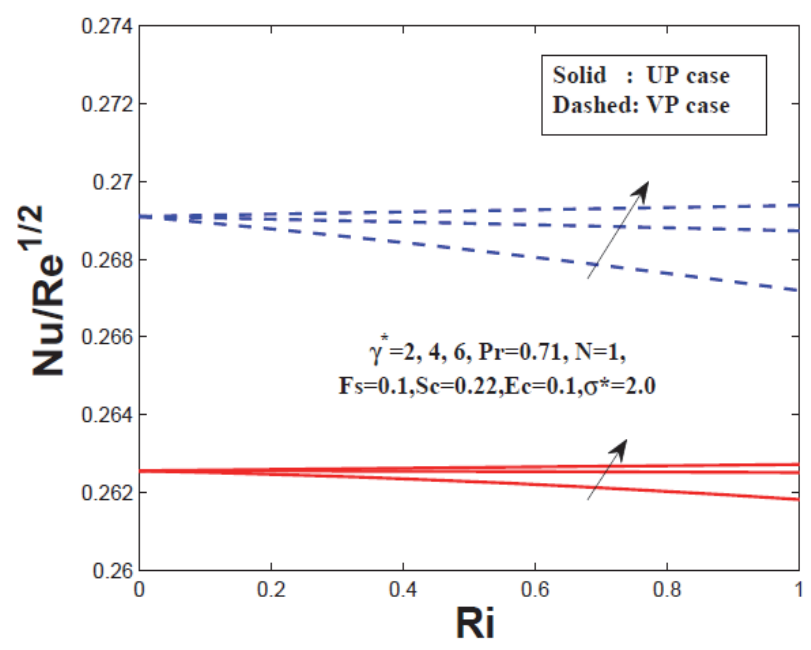

Fig. 24 Effect of $\gamma^{*}$ on heat transfer.

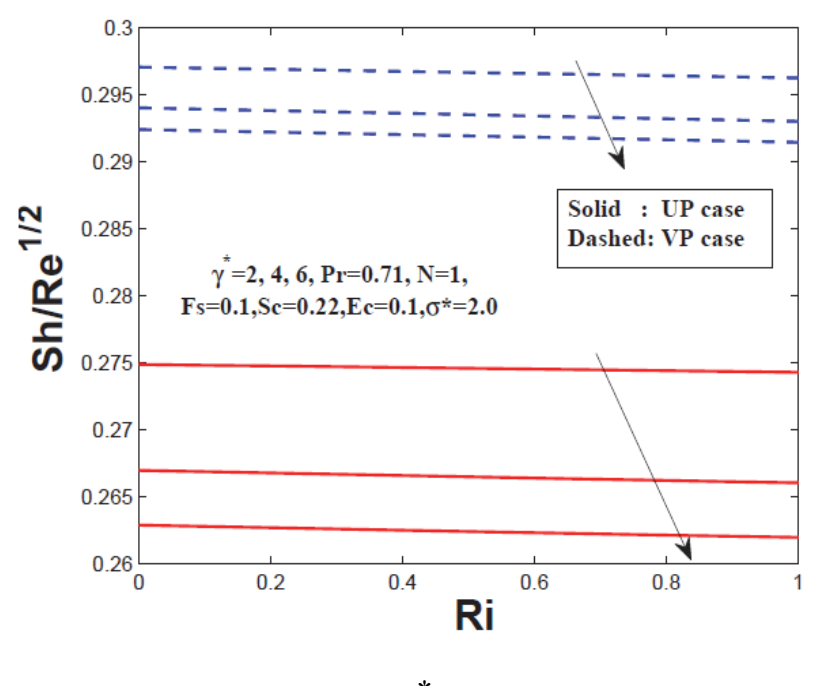

Fig. 25 Effect of $\gamma^{*}$ on mass transfer.

\section{CONCLUSIONS}

The following conclusions are drawn from the above numerical study:

- As the mixed convection parameter $R i$ increases, the velocity increases near the vertical plate, whereas its effect is reversed in the thermal and solutal boundary layer and also VP dominates over UP.

- As the Forchheimer number $F s$ increases, the velocity decreases, whereas the temperature and concentration profiles increases near the plate and also UP dominates over VP in the velocity and temperature.

- As the Prandtl number increases the velocity and temperature profiles decreases, but an opposite behaviour can be seen in the concentration profile.

- As increasing the Schmidt number $S c$, the velocity profile increases and the concentration profile decreases.

- As increasing thermal conductivity parameter, the velocity, temperature increases and is observed very less variation in the case of concentration.

- As increasing solutal diffusivity parameter, the velocity decreases, concentration increases and less variation is observed in the case of temperature.

\section{NOMENCLATURE}

$c_{b} \quad$ empirical constant

$T \quad$ temperature of the fluid

$T_{w} \quad$ temperature of the plate

$T_{\infty} \quad$ temperature far away from the plate

$k \quad$ thermal conductivity

$C_{P} \quad$ specific heat at constant pressure

$C \quad$ concentration of the fluid

$C_{w} \quad$ concentration of the plate

$C_{\infty} \quad$ concentration far away from the plate

$f \quad$ dimensionless velocity

$\eta \quad$ dimensionless similarity variable

Sh dimensional Sherwood number

$\mathrm{Nu}$ dimensional Nusselt number

$D_{m} \quad$ mass diffusivity

$P \quad$ Pressure

$k_{T} \quad$ thermal diffusion ratio

$q_{w} \quad$ average Nusselt number

$q_{m} \quad$ average Sherwood number

$\theta$ dimensionless temperature

$\phi \quad$ dimensionless concentration

$x \quad$ co-ordinate axis parallel to the plate

$y \quad$ co-ordinate axis perpendicular to the plate

Greek Symbols

$\beta_{T} \quad$ coefficient of thermal expansion

$\beta_{C} \quad$ coefficient of solutal expansion

$\rho \quad$ Density

$\bar{\mu} \quad$ effective Viscosity

$v \quad$ kinematic Viscosity

$\alpha \quad$ thermal diffusivity variation parameter

$\beta \quad$ coefficient of volume expansion

$\mu \quad$ viscosity

$\varepsilon \quad$ Porosity variation parameter

$\sigma \quad$ permeability parameter

$\tau \quad$ Skin friction

$\alpha^{*} \quad$ ratio of viscosities 


\section{Subscripts \\ $w \quad$ condition at the plate \\ $\infty \quad$ Condition far away from the plate}

\section{REFERENCES}

Ali J. Chamkha, Jasem, Al-Humoud, M.A. 2007, "Mixed Convection Heat and Mass Transfer of Non-Newtonian Fluids from a Permeable Surface Embedded in a Porous Medium," Int. Journal of Numerical Methods for Heat \& Fluid Flow, 17, 195 - 212. https://doi.org/10.1108/09615530710723966

Benenati, R.F., and Brosilow, C.B., 1962, "Void Fraction Distribution in Beds of Spheres," AICHE Journal, 8, 359-361.

https://doi.org/10.1002/aic.690080319

Chandrasekhara, B.C., and Namboodiri, P.M.S., 1985, "Influence of Variable Permeability on Combined Vertical Surfaces in Porous Medium," International Journal of Heat and Mass Transfer, 28, 199206.

https://doi.org/10.1016/0017-9310(85)90022-5

Hady, F.M., Bakier, A.K., and Gorla, R.S.R., 1996, "Mixed Convection Boundary Layer Flow on a Continuous Flat Plate with Variable Viscosity," Heat and Mass Transfer, 31, 169-172.

https://doi.org/10.1007/s002310050041

Harish Babu, D., and Satya Narayana, P.V., 2013, "Influence of Variable Permeability and Radiation Absorption on Heat and Mass Transfer in MHD Micropolar Flow over a Vertical Moving Porous Plate," Hindawi Publishing Corporation ISRN Thermodynamics. https://doi.org/10.1155/2013/953536

Hong, J.T., Yamada, Y., and Tien, C.L., 1987, "Effect of Non-Darcian and Non-Uniform Porosity on Vertical Plate Natural Convection in Porous Media," International Journal of Heat Mass Transfer, 109, 356-382.

https://doi.org/10.1115/1.3248088

Kafoussias, N.G., 1990, "Local Similarity Solution for Combined FreeForced Convective and Mass Transfer Flow past a Semi-Infinite Vertical Plate,” Int. J. Energy Res., 14, 305-309.

https://doi.org/10.1002/er.4440140306

Mehta, K.N., and Rao, K.N., 1994, "Buoyancy-Induced Fow of NonNewtonian Fuids in a Porous Medium Past a Vertical Plate With NonUniform Surface Heat Fux," Int. J. Eng. Sci., 32, 297-302.

https://doi.org/10.1016/0020-7225(94)90009-4

Merkin, J. H., 1972, "The Effect of Buoyancy Forces On the Boundary Layer Flow over a Semi-Infinite Vertical Flat Plate in a Uniform Free Stream," J. of Fluid Mech., 35, 439-450.

https://doi.org/10.1017/S0022112069001212.

Mohammadein, A.A., and El-Shaer, N.A., 2004, "Influence of Variable Permeability on Combined Free and Forced Convection Flow Past a Semi-Infinite Vertical Plate in a Saturated Porous Medium," Heat

Mass Transfer, 40, 341-346.

https://doi.org/10.1007/s00231-003-0430-3

Nalinakshi, N., Dinesh, P.A. and Chandrashekar, D.V., 2013, "Effects of Variable Fluid Properties and MHD on Mixed Convection Heat
Transfer from a Vertical Heated Plate Embedded in a Sparsely Packed Porous Medium," IOSR Journal of Mathematics, 7, 20-31. https://doi.org/10.9790/5728-0712031

Nield, D.A., and Bejan, A., 2013, "Convection in Porous Media," 4th Edition, Springer verlag, New York.

Pal, D., and Shivakumara, I.S., 2006, "Mixed Convection Heat Transfer From a Vertical Heated Plate Embedded in a Sparsely Packed Porous Medium," Int. J. Appl. Mech. Eng., 1, 929-939.

Rushi Kumar, B. and Shivaraj, R., 2013, "Heat and Mass Transfer in MHD Viscoelastic Fluid Flow over a Vertical Cone and Flat Plate With Variable Viscosity," Int. Journal of Heat and Mass Transfer, 56, 370379. https://doi.org/10.1016/j.ijheatmasstransfer.2012.09.001

Satya Narayana, P.V. and Sravanthi, S., 2012, "Influence of Variable Permeability on Unsteady MHD Convection Flow past a Semi Infinite Inclined Plate with Thermal Radiation and Chemical Reaction," Journal of Energy, Heat and Mass Transfer, 34, 141-161.

Satya Narayana, 2015, "Effects of Variable Permeability and Radiation Absorption on MHD Mixed Convective Flow in a Vertical Wavy Channel With Traveling Thermal Waves," Propulsion and Power Research, 4, 150-160.

https://doi.org/10.1016/j.jppr.2015.07.002

Schwartz, C.E., and Smith, J.M., 1953, "Flow Distributions in Packed Beds," Ind. Eng Chem. 45,1209-1218.

https://doi.org/10.1021/ie50522a025

Seddeek, M.A., 2004, "Thermal-Diffusion and Diffusion Thermo Effects on Mixed Free-Forced Convective Flow and Mass Transfer Over an Accelerating Surface with a Heat Source in the Presence of Suction and Blowing in the Case of Variable Viscosity," Acta Mechanica, 172, 83-94.

https://doi.org/10.1007/s00707-004-0139-5

Srinivasacharya, D., Mallikarjuna, B., and Chandrashekara, G., 2015, "Convective Heat Transfer Flow along a Sinusoidal Wavy Surface in a Porous Medium with Variable Permeability," Procedia Engineering, 127, 524-530.

https://doi.org/10.1016/j.proeng.2015.11.340

Subhas Abel, M. Siddheshwar, P.G., and Mahesha, N., 2009, "Effects of Thermal Buoyancy and Variable Thermal Conductivity on MHD Flow and Heat Transfer in a Power-Law Fluid over a Vertical Stretching Sheet in the Presence of a Non-Uniform Heat Source," Int. Journal of non-linear mechanics, 44, 1-12. https://doi.org/10.1016/j.ijnonlinmec.2008.08.002

Tierney, J.W., Roblee, L.H.S., and Barid, R.M., 1958, "Redial Porosity Variation in Packed Beds," AICHE Journal, 4, 460-464.

https://doi.org/10.1002/aic.690040415

Venkateswarlu, B., and Satya Narayana, P.V., 2016, "Influence of Variable Thermal Conductivity on MHD Caisson Fluid Flow Over a Stretching Sheet With Viscous Dissipation, Soret and Dufour Effects, Frontiers in Heat and Mass Transfer, 7-16.

https://doi.org/10.5098/hmt.7.16 